\title{
COVID-19: ENTRE EL DERECHO A LA SALUD Y LA PROTECCIÓN DE LA PRIVACIDAD
}

\author{
ANGELO VENCHIARUTTI \\ Università degli Studi di Trieste, Trieste, Italia \\ Recibido: 2/8/2020 - Aprobado: 9/8/2020 \\ doi: https://doi.org/10.26439/iusetpraxis2020.n50-51.5027
}

\begin{abstract}
RESUMEN. Mantener la privacidad del paciente se convierte en un dilema ético y legal para los médicos y los hospitales cuando ello puede causar daño a terceros. Este breve artículo trata de analizar las preguntas que surgen al respecto, con referencia a los pacientes con COVID-19. Para tal fin, teniendo en cuenta las directrices jurisprudenciales y doctrinales desarrolladas también en sistemas jurídicos distintos del italiano, se analizan las circunstancias que justifican la violación de la confidencialidad del paciente por parte de los médicos.
\end{abstract}

PALABRAS CLAVE: confidencialidad / médico / paciente / datos sanitarios / terceros / deber de proteger

\section{COVID-19: BETWEEN THE RIGHT TO HEALTH AND THE PROTECTION OF PRIVACY}

ABSTRACT. Keeping patient privacy becomes an ethical and legal dilemma for doctors and hospitals when this can cause harm to third parties. This short article aims to study the questions that arise in this regard with reference to patients with COVID-19. To this end, taking into account the jurisprudential and doctrinal guidelines also developed in legal systems other than the Italian, the circumstances that justify the violation of patient confidentiality by doctors are analyzed.

KEYWORDS: confidentiality / doctor / patient / health data / third parties / duty to protect 


\section{CONFIDENCIALIDAD Y EMERGENCIA EPIDEMIOLÓGICA DEL COVID-19}

La emergencia epidemiológica del COVID-19 ha sometido tanto a los profesionales como a las instituciones sanitarias a un esfuerzo significativo y encomiable en las intervenciones destinadas a tratar y contener la epidemia, especialmente en áreas en las que la infección se ha manifestado con particular acentuación.

En ausencia de terapias específicas, y a la espera de los resultados de la investigación sobre la posible vacuna, las medidas destinadas a contrarrestar la propagación del virus se han centrado, además de la hospitalización de los infectados en las estructuras hospitalarias, en el confinamiento voluntario de las personas y en imponer cuarentena a los infectados. Medidas que, evidentemente, han planteado una serie de problemas también en términos de protección de la confidencialidad en la relación médico-paciente. El problema se puede resumir de la siguiente manera: junto con el respeto a la confidencialidad de los infectados, puede haber necesidades opuestas. En general, todos los pacientes tienen derecho a la privacidad y la expectativa razonable de que la confidencialidad de su información personal será mantenida con rigor por parte de los profesionales sanitarios ${ }^{1}$. Empero, por otra parte, en algunos casos, la divulgación de la información confidencial -o su uso- es deseable o necesaria.

En cuanto a los pacientes con COVID-19, la situación radica en que, debido al obvio efecto estigmatizador que está destinado a ocurrir en las personas infectadas, los pacientes esperan que los médicos mantengan la confidencialidad de la información sobre su salud. También hay necesidades opuestas. Nos referimos en particular a la necesidad de prevenir el contagio al requerir la adopción de las precauciones imprescindibles para evitarlo, así como a la necesidad de informar a aquellos que pueden encontrarse en riesgo (miembros de la familia, personal de enfermería o custodia, voluntarios, etcétera) por haber entrado en contacto con la persona infectada.

Obviamente, el problema no surge si la parte interesada da su consentimiento para la comunicación de la información que le concierne. Pero ¿qué sucede si la parte interesada no da su consentimiento o se opone a la divulgación?

La emergencia del COVID-19 ha llevado al Gobierno italiano y a las autoridades de protección civil (como se ha llevado a cabo en otros países) a elaborar regulaciones destinadas a permitir, incluso en ciertas circunstancias, el control o la difusión de datos relacionado con personas consideradas en riesgo epidemiológico:

1 En Italia, una obligación específica de secreto para algunas figuras profesionales de no divulgar o publicitar información de la que tienen conocimiento por razones de trabajo deriva del artículo 622 del Código Penal. En cuanto a los médicos, la obligación de mantener en secreto se establece en el artículo 10, "Secreto profesional", del Código de Deontología Médica (2018). Sobre el tema, véase Barni (2011, p. 161). 
Con respecto a la relación médico-paciente, se puede inferir una imagen general de la documentación disponible en el sitio web del Garante para la Protección de Datos Personales. En particular, se han publicado una serie de respuestas a preguntas frecuentes (FAQ) que resumen y sistematizan las indicaciones dadas hasta ahora para el manejo correcto de los datos personales durante el periodo de emergencia. (Garante per la Protezione dei Dati Personali, s. f.)

Las preguntas frecuentes se dividen en cinco áreas temáticas. Entre las más relevantes para el tema que se trata, evidentemente están las incluidas en la categoría "Procesamiento de datos en el contexto sanitario en el ámbito de una emergencia sanitaria".

En primer lugar, debe recordarse que se afirma la legalidad del profesional sanitario durante la ejecución del hisopado del COVID-19 para preguntar al paciente la identidad de la persona positiva con la que mantuvo un contacto cercano, esto con el fin de reconstruir la cadena de los contactos que la persona diagnosticada con COVID-19 ha tenido. No hay indicaciones respecto a la legitimidad del profesional sanitario para comunicar el resultado positivo del hisopado a las personas con las que la infectada está destinada a mantener "contactos cercanos".

Por otro lado, se afirma que no hay nada que impida que el hospital donde se produjo la muerte de la persona infectada comunique a la compañía funeraria el estado positivo del fallecido por COVID-19 (dado que las disposiciones adoptadas durante la emergencia epidemiológica por coronavirus han previsto que, en casos de sospecha o muerte confirmada por dicha enfermedad, los operadores de servicios funerarios deben tomar precauciones especiales, similares a las ya previstas para la muerte de personas con enfermedades infecciosas y difusas, para evitar una infección adicional).

El Garante para la Protección de Datos Personales también se ocupa de la delicada cuestión relacionada con el suministro de información a los miembros de la familia, por parte de las instituciones sanitarias, sobre el estado de salud de los pacientes de COVID-19 que no pueden comunicarse de forma independiente. Al respecto, el Garante para la Protección de Datos Personales especifica que, en tales contextos, no existe ningún obstáculo para que el centro hospitalario asigne un número gratuito para comunicar tal información y proporcione medidas adecuadas para identificar a las personas que realmente tienen derecho a conocer sobre el estado de salud del familiar hospitalizado.

Por lo tanto, de esta aclaración se puede inferir el mantenimiento del derecho a la privacidad del paciente de COVID-19. Y esta circunstancia se confirma con la respuesta a la siguiente pregunta: ¿es posible difundir los datos de identificación de las personas positivas a COVID-19 o que han sido colocadas en aislamiento domiciliario? Al respecto, el Garante para la Protección de Datos Personales ha recordado que 
la legislación actual prohíbe la difusión de datos sensibles. Esta prohibición no fue anulada por la legislación de emergencia sobre la emergencia epidemiológica de COVID-19. Por lo tanto, las instituciones sanitarias y cualquier otra entidad pública o privada no pueden difundir, a través de sitios web u otros canales, los nombres de los casos verificados de COVID-19 o de los sujetos a la medida de aislamiento con el fin de contener la propagación de la epidemia. (s. f.)

Además, algunas de las disposiciones adoptadas por el Gobierno en vista de la contención de la pandemia parecen tener repercusiones en el tema de la confidencialidad. Piénsese en la disciplina de "medida de cuarentena con vigilancia activa". La disciplina establece que las "autoridades de salud territorialmente competentes" la adoptan (en particular) para "individuos que han tenido contactos cercanos con casos confirmados de enfermedades infecciosas propagadas por COVID-19" (Ministero della Salute, 2020). Para activar la cuarentena, presumiblemente será necesario informar a la parte interesada de la razón por la cual está sujeta a una medida que limita su libertad personal (durante al menos catorce días). Por lo tanto, se tratará de comunicarle, entre otros asuntos, la identidad del "caso confirmado" de COVID-19 con el que tuvo contactos cercanos.

Incluso, en ausencia de más estudios, la imagen obtenida de los datos que se han examinado hasta ahora parece estar algo fragmentada. Para intentar otorgar una respuesta a la pregunta formulada al principio de este breve ensayo, parece apropiado considerar otras situaciones. En el pasado, de hecho, ha habido casos en los que, en el contexto de una relación terapéutica, ha surgido un peligro para un tercero, y el médico se ha enfrentado al siguiente dilema delicado: preservar la confidencialidad del paciente o, en su lugar, informar a la persona amenazada para protegerla.

\section{¿CUÁNDO SE PUEDE REVELAR EL SECRETO? EL DEBER DE PROTEGER DEL PSICOTERAPEUTA}

Por lo tanto, parece apropiado mirar más allá de nuestras fronteras nacionales. La brevedad de esta contribución permite realizar solo unas pocas notas sobre la experiencia comparativa. Algunas ideas interesantes pueden inferirse de la evolución de algunos principios generales que han madurado en otras jurisdicciones con respecto a la relación entre el médico y el paciente.

En el contexto anglosajón, el principio según el cual el deber de confidencialidad del médico hacia el paciente no es absoluto también se encuentra bien arraigado. En ciertas circunstancias, el interés en la divulgación puede ser mayor que el interés en mantener la confidencialidad. 
Las cuestiones controvertidas surgen sobre todo en los campos psicológicos y psiquiátricos. En ese momento, las decisiones de la Corte Suprema de California sobre el caso Tarasoff (1974 y 1976) causaron un gran alboroto, lo que impuso un nuevo deber al psicoterapeuta: informar de inmediato o, como la segunda de las decisiones que la Corte Suprema de California más tarde especificó, proteger a la víctima potencial a expensas de la obligación de confidencialidad².

Los hechos del caso se pueden resumir de la siguiente manera: Prosenjit Poddar, estudiante de la Universidad de California, Berkeley, confió a su terapeuta —empleado de la referida universidad-que tenía la intención de tomar un arma y matar a la mujer de la que estaba enamorado, Tatiana Tarasoff. El terapeuta informó a la policía del campus de la amenaza. Pero ni la policía ni el terapeuta advirtieron a la joven directamente. Cuando esta regresó de vacaciones, Poddar la mató.

Los padres de Tatiana demandaron a la policía del campus y a los Regents of University of California (administración) por no advertir a su hija. La Corte Suprema de California, en una primera decisión (Tarasoff v. Regents of University of California, 1974), dictaminó que los terapeutas tenían el "duty to warn others who are in foreseeable danger from the therapists' patients". Y en una segunda decisión (Tarasoff v. Regents of University of California, 1976) el tribunal atenuó el deber de advertir en un deber de proteger, entendido como el deber "to use reasonable care to protect the intended victim against such danger".

Las dos decisiones de la Corte Suprema de California no han dejado de suscitar críticas y reservas dentro de la comunidad médica, así como entre los estudiosos del derecho (Klinka, 2009) ${ }^{3}$. En particular, se ha objetado que la simple relación entre el médico y el paciente no parece suficiente para justificar la existencia de tal deber para los psicoterapeutas. En el caso, la relación entre el psicoterapeuta y el estudiante era demasiado débil como para configurar respecto al primero el deber de controlar la conducta del segundo (Harper y Kime, 1934, p. 898).

En todo caso, frente a las críticas, los dos pronunciamientos no han quedado aislados. Los tribunales de los Estados Unidos de América han aplicado las reglas desarrolladas en el caso Tarasoff, aunque con una cierta variedad de avisos. Además, en la mayoría

2 Las dos decisiones de Tarasoff fueron fuertemente influenciadas por el artículo de Fleming y Maximoc (1974, p. 1025), publicado mientras el caso aún estaba pendiente ante la Corte Suprema de California. Para un comentario crítico de las dos decisiones, véase Stone (1976, p. 358).

3 Recuérdese que, ya después de las dos decisiones, más de un comentarista había propuesto varios efectos negativos de la Tarasoff Doctrine: en caso de violación de la confidencialidad, los pacientes que ya estaban en terapia se habrían sentido traicionados por su médico; dada tal eventualidad (es decir, la divulgación de lo que el paciente le había confiado al galeno), aquellos que aún no estaban en terapia habrían sido disuadidos de comenzarla. 
de los estados, regulaciones específicas han establecido el deber del psicoterapeuta de informar a la posible víctima de su paciente. Sin embargo, existen diferencias significativas entre una y otra legislación. Por lo tanto, sigue habiendo una gran incertidumbre en cuanto a las circunstancias en que los psicoterapeutas deben violar la confidencialidad e informar a las posibles víctimas de sus pacientes.

Al final de este párrafo, ha de tenerse en cuenta que el reconocimiento de una posición de garantía en el campo de la responsabilidad médica no es ajeno al sistema legal italiano. Surge del establecimiento de la relación terapéutica con el paciente. Y estas circunstancias a veces han llevado a los jueces italianos a creer que los psiquiatras tienen la obligación de proteger a terceros ${ }^{4}$.

\section{CONFIDENCIALIDAD DEL PACIENTE Y ENFERMEDADES CONTAGIOSAS}

Siempre en el entorno legal de los Estados Unidos de América, el aumento progresivo de las personas con síndrome de inmunodeficiencia adquirida (sida) también ha generado preguntas delicadas. En particular, más de un autor se preguntó si los médicos podrían ser responsables del contagio por no haber brindado información a terceros sobre el riesgo de contraer el virus del sida de portadores identificados (Baruch, 1987, p. 169).

Si bien se reconoce la importancia especial de respetar la confidencialidad del paciente en este contexto, varios estudiosos han destacado que el derecho a la privacidad no es absoluto. En específico, se ha señalado que el médico puede violar el deber de confidencialidad cuando la divulgación de la información confidencial parezca necesaria para prevenir la propagación de una enfermedad contagiosa. Estas son hipótesis a las que los jueces del caso Tarasoff ya se habían referido. De hecho, no faltaron quienes iniciaron acciones legales contra los médicos para buscar una indemnización por los daños sufridos por no ser informados de la enfermedad de la pareja 5 . Sin embargo, a lo largo de los años se han expresado fuertes reservas, tanto por parte de la comunidad médica como de la legal, sobre la posibilidad de extender el deber de advertir en el caso de los médicos que tratan a pacientes con sida. En cualquier caso, se ha observado que la obligación del médico ha de ceñirse a un número bastante limitado de personas: es decir,

4 Cass. pen. 11 marzo 2008, n. ${ }^{10795}$, en https://www.neldiritto.it, quien confirmó la sentencia del psiquiatra por la muerte de uno de los trabajadores sanitarios. Sobre el tema de la legislación psiquiátrica en Italia, permítasenos mencionar Venchiarutti (2020).

5 Es conocido el caso en el que el examante de Rock Hudson, Marc Christian, demandó por daños tanto a los herederos como a los médicos del actor. Christian se quejó de que no había sido informado de la enfermedad de Hudson. Aunque Christian no había mostrado ningún síntoma de la enfermedad, el juez de primera instancia decidió aceptar el reclamo por daños y perjuicios por intentional infliction of emotional distress. Como la cantidad liquidada fue menor a la solicitada, Christian apeló la decisión de primera instancia. En 1991, se llegó a un acuerdo extrajudicial entre las partes que terminó la disputa. Al respecto, véase Hermann y Burris (1993). 
las que - según el conocimiento del galeno- es probable que, debido a sus relaciones, contraigan la patología del paciente ${ }^{6}$.

También en Italia la propagación del sida y las crecientes preocupaciones relacionadas con la salud pública han estimulado un estudio en profundidad del tema relacionado con la naturaleza absoluta o relativa del secreto profesional (Casonato, 1996, p. 230). Para no dejar la solución de un asunto tan relevante y delicado a la responsabilidad del médico individual, el legislador ha brindado algunas indicaciones específicas:

La ley del 5 de junio de 1990, n. 135, sobre el tema de las intervenciones urgentes en el campo de la prevención y la lucha contra el SIDA. El artículo 5 establece, en el párrafo 5, que "los profesionales de la salud que, en el ejercicio de su profesión, se den cuenta de los casos de infección por VIH o SIDA, deben tomar todas las medidas necesarias para proteger la confidencialidad de la persona asistida", y, en el párrafo 4, que "la comunicación de los resultados de las pruebas de diagnóstico directas o indirectas para la infección por VIH solo puede darse a la persona a la que se refieren estas pruebas". Sin embargo, incluso si se refiere a un caso con una diferente enfermedad infecciosa (hepatitis C), el Tribunal Supremo de Italia ha considerado que el derecho a la salud física del tercero o de la comunidad prevalece contra el derecho a la defensa de los datos personales?

\section{INFORMACIÓN SOBRE ENFERMEDADES GENÉTICAS}

Incluso en el contexto de las enfermedades genéticas, los avances en la ciencia dificultan la resolución del conflicto entre el derecho al secreto y el de la información. Una decisión reciente del Tribunal Superior de Justicia de Inglaterra (Quens Bench Division) ha decidido sobre este tema.

El contexto en el cual comienza la sentencia del tribunal inglés está marcado por una serie de eventos trágicos e inusuales. El padre de la actriz $A B C$ había matado a la

6 Por ejemplo, en Simonsen v. Swenson, 104 Neb. 224, 177 N. W. 831 (1920), el Tribunal Supremo del Estado de Nebraska decidió que el médico puede revelar información confidencial de un paciente cuando sea necesario para evitar la propagación de una enfermedad contagiosa. Asimismo, en Davis v. Rodman, 147 Ark. 385, 227 S. W. 612 (1921), el Tribunal Supremo del Estado de Arkansas dictaminó que, al tratar a un paciente con una enfermedad contagiosa, el médico tiene el deber de advertir a los familiares de aquel. De nuevo, en Hofmann v. Blackmon, 241 So.2d 752 (Fla. Dist. Ct. App. 1970), cert. denied, 245 So. 2d 257 (Florida, 1971), la Florida District Court consideró que el médico, una vez que el paciente sea diagnosticado con una enfermedad contagiosa, tiene el deber de advertir a sus familiares.

7 El asunto se refería a la indemnización por el daño sufrido por el demandante tras haber contraído una enfermedad infecciosa grave (que luego condujo a su muerte), transmitida a él por su esposa. La mujer se enfermó por transfusiones de sangre infectada. Aunque los médicos conocían la grave patología, sintieron que tenían que proteger el derecho a la privacidad de los datos confidenciales. Por lo tanto, no proporcionaron ninguna comunicación a su esposo. En cambio, el tribunal consideró la prevalencia del derecho a la salud del esposo y de la comunidad sobre el derecho a la defensa de los datos personales. 
madre de la niña a disparos. Mientras estaba internado en algunos centros sanitarios, el hombre fue diagnosticado con la enfermedad de Huntington: un mal neurodegenerativo que se manifiesta con anormalidades en el movimiento, problemas cognitivos y síntomas psiquiátricos.

Después de un tiempo, el personal médico que estaba tratando al hombre se enteró de que $A B C$ estaba embarazada. Dicho personal conocía a la mujer: esta también había aceptado participar en algunas sesiones de psicoterapia familiar. En vista del alto riesgo de transmisión genética, los médicos tenían la intención de informar a la mujer sobre lo que le habían diagnosticado a su padre. Sin embargo, este no quería dar su consentimiento y, por lo tanto, no se comunicó nada a ABC. Solo después de unos años, la mujer descubrió que sufría la misma patología genética que su padre. Las pruebas médicas también revelaron la probable progresión degenerativa de la enfermedad en unos pocos años. Además de estar angustiada por su situación, la mujer estaba profundamente preocupada por su hijo.

Posteriormente, $A B C$ demandó a los tres centros sanitarios en los que su padre había sido hospitalizado y pidió una indemnización por el daño psíquico sufrido y por los daños posteriores como consecuencia. Según la mujer, también debido a su embarazo, los tres centros sanitarios habían violado el deber de cuidarla por no haberle advertido sobre el riesgo de transmisión del gen que causa la enfermedad de Huntington.

La demandante señaló primero que los médicos eran conscientes del hecho de que ella sería una madre soltera, la única responsable de la educación y el cuidado de su hijo. Agregó que, si hubiera sido informada del diagnóstico realizado a su padre, se habría sometido a una prueba de inmediato para determinar la presencia de la enfermedad de Huntington. Y la mujer afirmó que, si la prueba hubiera confirmado la existencia de dicha enfermedad, habría interrumpido el embarazo para no exponer al niño al riesgo de depender (en el futuro) de una madre soltera gravemente enferma, o de convertirse en un huérfano en breve tiempo o de heredar la enfermedad. En este caso específico, los médicos y otros profesionales sanitarios debían haber considerado prevalentes sus derechos e intereses sobre los de proteger la privacidad de su padre.

La Cour of Appeal de Inglaterra había anulado una primera decisión que rechazaba las reclamaciones de la mujer. Luego vino el pronunciamiento de la High Court. La jueza reconoció que algunos profesionales de la salud (especialmente los que organizaron la psicoterapia familiar) tenían la obligación legal de equilibrar los derechos e intereses de sus pacientes para preservar su confidencialidad con los de otra persona, en que la divulgación de la salud del paciente podía reducir o prevenir un riesgo significativo de daño grave para el tercero. 
Sin embargo, respecto al caso específico, para la jueza Yip, los profesionales sanitarios habían equilibrado, según un estándar razonable, los intereses opuestos del paciente y los de la hija. Así, la mencionada jueza concluyó lo siguiente:

I have not found any actionable breach of duty on the part of the second defendant. The decision not to disclose was supported by a responsible body of medical opinion and was a matter of judgment open to the second defendant after balancing the competing interests.

La decisión fue inmediatamente considerada significativa por los comentaristas en más de un aspecto. En primer lugar, porque establece por primera vez la existencia en el common law de un legal duty of care para un médico u otros profesionales sanitarios: este duty of care no encuentra su origen exclusivamente en reglas éticas, y estaría dirigido a equilibrar el derecho del paciente a garantizar su confidencialidad con los intereses públicos y los derechos de terceros en riesgo.

Como se desprende de la breve descripción del caso, tal deber surge en presencia de circunstancias singulares. Implica, sobre todo, la existencia de una relación cercana entre la persona interesada en recibir la información y el médico. En el caso examinado por el tribunal superior, la situación específica de $A B C$ era bien conocida por uno de los equipos médicos - el que había promovido las sesiones de psicoterapia familiar-.

Según el tribunal, también es necesario determinar que existe una probabilidad significativa de que el tercero pueda sufrir daños si no se le informa. $Y$, en el caso concreto -especificó el tribunal-, $A B C$ estaba en peligro de sufrir daños si la información sobre su riesgo genético no se había proporcionado; no solo era previsible, sino que los médicos del paciente lo habían previsto expresamente, tal como surgió durante el proceso.

La orientación jurisprudencial se alinea así con el puesto ya consolidado en el ámbito profesional. La jueza especifica que la obligación legal es paralela a la establecida en el campo profesional y debe ejercerse siguiendo las indicaciones del Consejo Médico General y las formuladas por las entidades en sectores especializados.

\section{ALGUNAS CONCLUSIONES}

Es momento de realizar algunas consideraciones concluyentes. La breve ilustración de los estudios de caso revela lo siguiente: de acuerdo con las reglas generales, el médico debe guardar el secreto de todo lo que el paciente le confía o que puede saber por su profesión. Sin embargo, en ciertas circunstancias, la divulgación de esa información puede estar justificada por intereses generales. En otras palabras, la divulgación de información confidencial a terceras personas podría ser justificable si se hace con el objeto de proteger derechos fundamentales de terceras partes o por un interés público 
legalmente protegido. La divulgación solo estará justificada en circunstancias excepcionales, es decir, si sirve a un interés que, en esas circunstancias en particular, pese más que el derecho del paciente a la privacidad.

Toda vez que contrapesar los derechos del paciente con otros derechos e intereses es siempre difícil, esto puede llevarse a cabo más fácilmente si el conflicto ocurre con los derechos de terceras partes identificables o con un interés público difuso, como la salud pública.

Volvamos al tema inicial de estas páginas. La situación de salud de emergencia de COVID-19 destaca la necesidad de equilibrar la protección de la privacidad de la persona enferma con las necesidades opuestas de rapidez y urgencia de la intervención para garantizar la salud (así como el paciente) de terceros. En las páginas iniciales, se ha recordado que el legislador italiano ha autorizado, con diversas medidas, la comunicación de los datos personales de las personas infectadas entre las diversas instituciones competentes en el sector de la salud para hacer frente a la emergencia de COVID-19. De hecho, algunas de esas medidas establecen que la comunicación de los datos personales también se brinde a sujetos públicos y privados que no sean instituciones sanitarias. En algunos casos, entonces, el médico y las instituciones sanitarias se encuentran en la obligación de informar a terceros sobre las condiciones de salud de la persona infectada. Esto se refiere a los casos en que las autoridades sanitarias competentes deben adoptar la medida de "cuarentena con vigilancia activa" contra "personas que han tenido contactos cercanos con casos confirmados de enfermedad infecciosa difusa COVID-19".

Se solicitaron acciones destinadas a regular orgánicamente las situaciones de emergencia provocadas por el coronavirus, sobre todo en lo que respecta a las medidas que limitan los derechos y las libertades fundamentales (Gatta, 2020). Por las circunstancias que se han examinado en este breve ensayo, las reglas éticas de los sectores profesionales interesados también han de actualizarse. Ya en la versión actual del Código Italiano de Deontología Médica hay algunas hipótesis sobre la causa justa de revelación para el médico de lo que le ha sido confiado o que ha podido saber debido a su profesión. Alguna sugerencia proviene de la experiencia extranjera. La articulación sobre el mismo aspecto de las directrices del Consejo Médico General -a las que se refirió expresamente la High Court de Inglaterra en la decisión antes mencionada- es bastante distinta. En ellas leemos lo siguiente:

Personal information may, therefore, be disclosed in the public interest, without patients' consent, and in exceptional cases where patients have withheld consent, if the benefits to an individual or society outweigh both the public and the patient's interest in keeping the information confidential. You must weigh the harms that are likely to arise from nondisclosure of information against the possible harm, both to the patient and to the overall trust between doctors and patients, arising from the release of that information. (ABC v St George's Healthcare NHS Trust \& Ors, 2020) 


\section{REFERENCIAS}

ABC v St George's Healthcare NHS Trust \& Ors. (28 de febrero del 2020). EWHC 455. Recuperado de https://www.bailii.org/ew/cases/EWHC/QB/2020/455.html

Barni, M. (2011). I doveri del medico. En S. Rodotà y P. Zatti (Dirs.) y L. Lenti, E. Palermo Fabris y P. Zatti (Eds.), Trattato di biodiritto. I diritti in medicina (pp. 139-171). Milán: Giuffrè.

Baruch, D. W. (1987). AIDS in the Courts: tort liability for the sexual transmission of acquired immune deficiency syndrome. Tort \& Insurance Law Journal, 22(2), 165-193.

Casonato, C. (1995). Diritto alla riservatezza e trattamenti sanitari obbligatori: un'indagine comparata. Università degli Studi di Trento.

Fleming, J. y Maximoc, B. (1974). The patient or his victim: the therapist's dilemma. California Law Review, (62), 1025-1068. Recuperado de https://heinonline.org/ $\mathrm{HOL} /$ LandingPage?handle=hein.journals/calr62\&div=42\&id=\&page=

Garante per la Protezione dei Dati Personali. (s. f.). COVID-19 e protezione dei data personali. Recuperado de https://www.garanteprivacy.it/temi/coronavirus/faq

Gatta, G. L. (2020). I diritti fondamentali alla prova del coronavirus. perché è necessaria una legge sulla quarantena. En Sistema Penale, 02 aprile. Recuperado de http:// www.ristretti.it/commenti/2020/aprile/pdf/articolo_gatta.pdf

Harper, F. V. y Kime, P. M. (1934). The duty to control the conduct of another. Yale Law Journal, (43), 886-905.

Hermann, D. H. J. y Burris, S. (1993). Torts: Private law suits about HIV. En S. Burris, H. L. Dalton y J. L. Miller (Eds.), AIDS law today (pp. 341 y ss.). Yale University Press.

Ministero della Salute. (2020). Circolare: Misure urgenti per fronteggiare l'emergenza epidemiologica da COVID-19.

Stone, A. A. (1976). The Tarasoff decisions: suing psychotherapist to safeguard society. Harvard Law Review, (90), 358-378.

Tarasoff v. Regents of University of California. (1974). 529 P.2d 553, 118 Cal.

Tarasoff v. Regents of University of California. (1976). 551 P.2d 334, 131 Cal. Rptr.14.

Venchiarutti, A. (2020). Asistencia psiquiátrica y protección civil de la persona discapacitada: recurrencia y posibilidad de reforma. En C. A. Agurto Gonzales y S. L. Quequejana Mamani (Dirs.), Derecho privado y comparación jurídica. El aporte de la escuela jurídica italiana (pp. 135 y ss.). Santiago de Chile: Ediciones Olejnik. 


\section{BIBLIOGRAFÍA}

Cass. Civ. 11994/2017. (6 de mayo del 2017). Corte di Cassazione, Sez. III Civile, Sentenza. Recuperado de https://www.privacy.it/2017/05/06/cass-privacy-paziente-vssalute-terzi/

Hermann, D. H. J. (1986-1987). AIDS: Malpractice and transmission liability. Colorado Law Review, 58(1), 63-107.

Klinka, E. (2009). It's been a privilege: advising patients of the Tarasoff duty and its legal consequences for the federal psychotherapist-patient privilege. Fordham Law Review, (78), 863-931.

Talbot. J. S. (1988). The conflict between a doctor's duty to warn a patient's sexual partner that the patient has AIDS and a doctor's duty to maintain patient confidentiality. Washington and Lee Law Review, (45), 355-380.

Thornton, J. (2020). Judgment in ABC case rules on confidentiality. The Lancet, (395), 771-772. doi:10.1016/S0140-6736(20)30494-3 